\title{
Uma alternativa mecânica com emprego de forças magnéticas para a desimpactação dentária
}

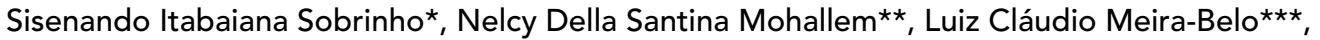

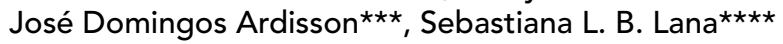

\section{Resumo}

Objetivo: o presente trabalho teve como finalidade avaliar clinicamente uma nova alternativa mecânica para a erupção guiada ao plano oclusal de um dente pré-molar impactado empregando magnetos atrativos da liga samário-cobalto. Metodologia: o tratamento cirúrgico-ortodôntico foi realizado em uma paciente jovem de 11 anos portadora de oclusão de Classe I de Angle, com impactação do segundo pré-molar inferior direito. O sistema magnético empregado consistiu de um magneto colado com resina fotopolimerizável sobre a superfície vestibular do dente impactado e um outro pólo magnético rígido incrustado em um aparelho banda alça. Previamente à instalação do sistema magnético no meio bucal, os magnetos foram caracterizados através de análise de fluorescência, difração de raios X e nível de magnetização. A relação força/distância empregada foi quantificada, os magnetos foram medidos e a geometria magnética bucal estabelecida. Resultados: o tempo empregado na desimpactação foi de 40 dias e foram necessárias duas ativações magnéticas por aproximação dos magnetos neste intervalo. A opção magnética neste caso clínico foi bastante eficaz tanto em relação ao tempo de tratamento quanto em relação ao conforto proporcionado ao paciente. Os magnetos empregados geraram um campo de força contínuo e autônomo não sendo necessária a utilização de fios metálicos como guias de orientação na erupção e nem elásticos de tracionamento. Conclusão: esta possibilidade terapêutica poderá ser útil em desimpactações mais profundas tais como os caninos ectópicos, cuja ocorrência é freqüente.

Palavras-chave: Forças magnéticas. Ortodontia. Desimpactação dentária.

\section{INTRODUÇÃO}

Um dente impactado é aquele que, na época normal de erupção, fica retido no interior do osso, não irrompendo ${ }^{11}$. Esta anomalia se localiza prin- cipalmente nos caninos e terceiros molares ${ }^{14}$.

Em um estudo sobre a incidência de dentes inclusos, em uma amostra de 3.000 pacientes, Verdi et al. ${ }^{24}$ observaram que $8 \%$ (245 pacientes)

* Ortodontista. Mestrando em Engenharia de Materiais REDEMAT. Pesquisador Associado do departamento de Química da UFMG.

** Professora Adjunta do Departamento de Química ICEX, Universidade Federal de Minas Gerais. Doutora em Física Aplicada.

*** Pesquisador do Centro de Desenvolvimento da Tecnologia Nuclear CDTN/CNEM. Doutor em Física pela UFMG.

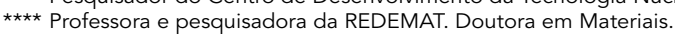


eram portadores de impactações dentárias. Nesta amostra foram encontrados 360 dentes inclusos, dos quais a maior incidência de dentes impactados foi nos molares, com $58.6 \%$, seguido dos caninos com $18.9 \%$ e supra numerários $11.4 \%$. Os pré-molares foram responsáveis por $8 \%$ das impactações e os incisivos 3\%. Apesar da literatura não ser conclusiva no que concerne à etiologia das impactações dentárias, tem-se sugerido um enfoque multifatorial envolvendo fatores locais e sistêmicos. Andreasen ${ }^{1}$, por exemplo, citou alguns obstáculos como o comprimento deficitário da arcada dentária, ectopia do germe dentário, anquilose do primeiro molar decíduo, presença de tecido fibroso sobre o dente, presença de dentes supranumerários e odontomas como fatores locais. Fatores sistêmicos e genéticos também contribuem para desordens eruptivas, além da displasia cleidocraniana ${ }^{19}$, sífilis, tuberculose e anemias ${ }^{11}$. Nomura et al. ${ }^{17}$ acrescentaram ainda como fatores etiológicos as inflamações do saco folicular durante a erupção dentária, traumas e estimulações durante a formação dentária e desordens endócrinas.

Bianchi e Roccuzzo ${ }^{3}$ classificaram as impactações em primárias e secundárias, referindo-se às dentições decíduas e permanentes respectivamente. Distúrbios de erupção na dentição decídua (primária) podem ser considerados raros. Entretanto, as impactações secundárias são amplamente observadas clinicamente.

Muitos termos são empregados para definir esta anomalia: depressão, submersão, hipoprotusão, infraoclusão. Todos estes termos tentam descrever o posicionamento dentário em relação ao plano oclusal ${ }^{12}$.

Antropologicamente, as impactações dentárias e a hipodontia são consideradas alterações evolucionárias e estes problemas estão presentes no homem moderno. Entretanto, a descoberta de um canino impactado em um crânio em Vukovar na Croácia, com idade de $2700 \mathrm{AC}$, indica que a impactação também ocorria no homem antigo e tem acompanhado o homem por milhares de anos. Parece então provável que esta anormalidade na erupção dos dentes humanos não seja causada por condições modificadas trazidas pela civilização moderma $^{18}$.

Com o avanço da Odontologia, nas últimas décadas, nos aspectos de diagnóstico, biomateriais e procedimentos clínicos, as impactações dentárias ganharam uma nova concepção diagnóstica e terapêutica. Uma abordagem conservadora que objetiva inserir estes dentes impactados funcionalmente nas arcadas dentárias só é possível numa visão integradora e multidisciplinar. Para isso é necessário que se conheça as diversas alternativas propostas na literatura, enfatizando as definições, os graus de incidência, os fatores etiológicos e as condutas terapêuticas propostas.

Dentre as possibilidades clínicas de desimpactação dentária, a erupção guiada pelo método de tracionamento cirúrgico-ortodôntico parece ser a mais eficaz ${ }^{4}$. Este procedimento pode ser realizado através da exposição cirúrgica da coroa do dente em questão e pelo seu laçamento com fios de aço, transfixação da coroa ou pela fixação direta de dispositivos ortodônticos com resinas, permitindo assim a aplicação de uma força ${ }^{4}$.

O emprego de fios flexíveis circunferenciais ao redor do colo dentário foi uma prática bastante comum no passado. No entanto, esse procedimento além de ser invasivo, pois remove uma grande quantidade óssea, pode acarretar anquilose e reabsorções externas ${ }^{7}$. McDonald e Yap ${ }^{16}$, buscando um maior controle de ancoragem, desimpactaram dentes com emprego de colagem direta de braquetes sobre a coroa, porém apoiaram os seus elásticos sobre placas acrílicas. Majourau e Norton $^{13}$ desimpactaram o primeiro molar inferior através de colagem direta de um botão ortodôntico sobre a coroa do dente, empregando molas distalizadoras. Shapira et al. ${ }^{20}$ desimpactaram segundos pré-molares inferiores que se encontravam bastante inclinados distalmente. Após exposição cirúrgica, os dentes envolvidos receberam braquetes 
colados com resina e foram tracionados por forças elásticas, apoiados em uma aparatologia fixa.

Embora todas as alternativas cirúrgico-ortodôntico empregadas para guiar os dentes impactados à sua normalidade funcional tenham logrado êxito, novas propostas terapêticas com novos materiais têm surgido nos últimos anos, na Ortodontia. O emprego de magnetos miniaturizados atrativos é um dos processos mais recentes na desimpactação dentária guiada, sendo o procedimento associativo cirúrgico-ortodôntico.

Forças magnéticas têm sido incorporadas à mecânica ortodôntica durante os últimos anos ${ }^{22}$. A inovação no uso dos magnetos em Odontologia veio com a introdução de novas ligas magnéticas. Estes magnetos terra raras que pertencem à família dos lantanídeos, como as ligas de samáriocobalto $\left(\mathrm{SmCo}_{5}, \mathrm{Sm}_{2} \mathrm{Co}_{17}\right)$, são 20 vezes mais fortes que os permanentes anteriores, o alumínioníquel-cobalto $\left(\mathrm{AlNiCo}_{5}\right)$. Estudos extensos têm demonstrado que os magnetos lantanídeos têm um bom nível de biocompatibilidade ${ }^{6,8}$. Em termos físicos, as novas ligas magnéticas se caracterizam por uma alta força coercitiva $(\mathrm{H})$, que significa capacidade do magneto ser desmagnetizado sob a influência de um campo magnético; alta resistência (B), ou indução magnética, que indica a extensão da magnetização espontânea; e um alto produto energético $(\mathrm{BxH})$, que indica a capacidade de atração e repulsão ${ }^{2}$. Convém salientar que estes magnetos de samário-cobalto apresentam uma temperatura Curie, temperatura no qual um magneto permanente inicia irreversivelmente a perda de suas propriedades magnéticas, de $350^{\circ} \mathrm{C}$, o que propicia a esterilização dos mesmos sem perdas de suas propriedades possibilitando a sua reutilização clínica.

Clinicamente esta opção ortodôntica foi inicialmente proposta por Springate e Sandler ${ }^{23}$, na desimpactação de pré-molares. O campo magnético instalado em configuração atrativa serviu de guia de erupção aos dentes impactados. Posteriormente Darendeliler, Friedli ${ }^{9}$ e Yuksel et al. ${ }^{25}$ tam- bém obtiveram êxitos em suas desimpactações de pré-molares e caninos, respectivamente, com o emprego de campos magnéticos atrativos.

\section{OBJETIVO}

O presente trabalho teve por objetivo demonstrar a utilização dos campos magnéticos atrativos gerados por magnetos permanentes da liga samário-cobalto $\left(\mathrm{Sm}_{2} \mathrm{Co}_{17}\right)$, previamente caracterizados magneticamente para a desimpactação do segundo pré-molar inferior direito em um paciente jovem.

\section{MATERIAL E MÉTODOS}

\section{Descrição do caso clínico}

O estudo foi realizado na arcada inferior de um paciente do gênero feminino, leucoderma, com 11 anos e 2 meses de idade. A paciente se encontrava em fase de dentição mista (Fig.1A).

Durante a avaliação clínica foi constatada a ocorrência de uma má oclusão de Classe I de Angle, com uma discreta mordida profunda, um perfil reto e um equilíbrio nas proporções da face. As condições gerais de higiene e saúde bucal eram satisfatórias e a paciente não apresentava história de nenhuma patologia local ou sistêmica. O histórico familiar mostrava um irmão mais velho que também apresentava retenção dos segundos prémolares inferiores.

As radiografias periapicais, ortopanorâmicas e a teleradiografia em norma lateral ratificaram a normalidade das bases ósseas, bem como a morfologia

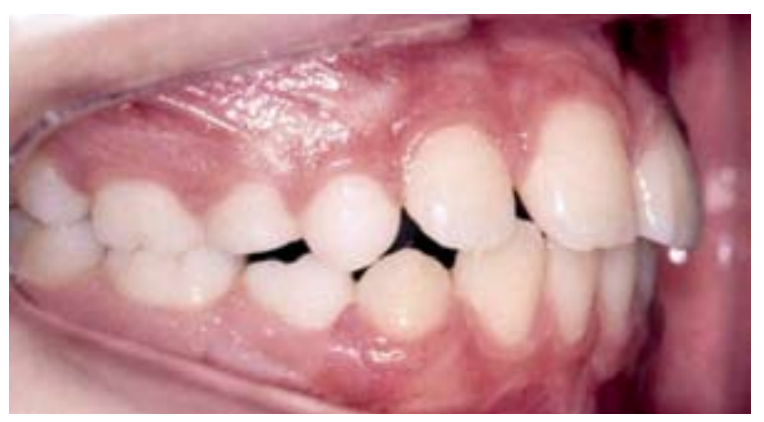

FIGURA 1A-Aspectos clínicos iniciais. 
dentária. No entanto, verificou-se a impactação do segundo pré-molar inferior direito junto à raiz mesial do primeiro molar inferior permanente direito (Fig. 1B), apresentando um estágio de rizogênese bastante atrasada. Verificou-se ainda uma inclinação coronária no sentido vestíbulo lingual (Fig. 2). $\mathrm{O}$ dente em questão posicionava-se inclinado em relação ao plano oclusal em $42^{\circ}$, conforme averiguação feita na radiografia cefalométrica (Fig.1B).

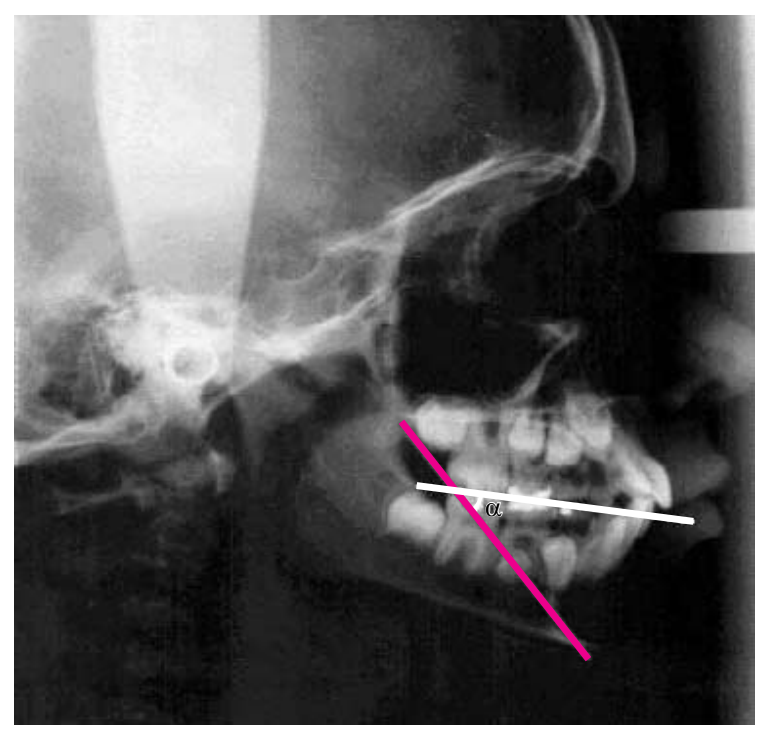
FIGURA 1B - Inclinação distal da coroa do segundo pré-molar inferior direito $\left(\alpha=42^{\circ}\right)$.

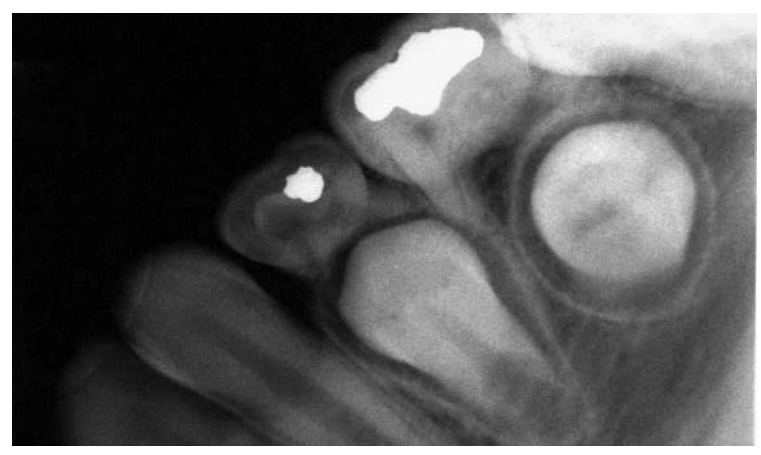

FIGURA 2 - Inclinação vestíbulo-lingual da coroa do segundo pré-molar inferior direito.

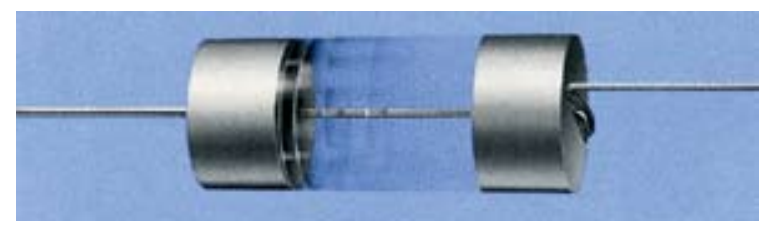

FIGURA 3 - Magnetos terra raras da liga samário-cobalto $\left(\mathrm{Sm}_{2} \mathrm{Co}_{17}\right)$.

\section{O sistema magnético}

O dispositivo magnético empregado foi confeccionado utilizando um par de magnetos da liga samário-cobalto cilíndricos com $\mathrm{h}=2,1 \mathrm{~mm}$, $\theta=3,7 \mathrm{~mm}$ e $\mathrm{m}=0.15 \mathrm{~g}\left(\mathrm{Ormco}^{\circledR}\right)$ revestidos previamente por um filme fino de parilene para evitar corrosão (Fig. 3). Este dispositivo foi previamente avaliado quanto aos níveis de força magnética em função das distâncias.

\section{Caracterização dos magnetos}

A composição química dos magnetos foi verificada através de uma análise de fluorescência e da difração de raios X. Foram realizados também testes de dureza (Vickers) e de magnetização.

Para uma melhor adequação da posição entre os magnetos, permitindo a manutenção da força magnética sem que houvesse inversão do campo, foram realizadas medições da variação da força gerada pelo campo magnético em função da variação da angulação intermagnetos.

\section{Instalação do dispositivo magnético}

Um dispositivo banda-alça foi preliminarmente elaborado em um modelo de gesso. Em seguida foi acrilizado em sua superfície oclusal com a finalidade de reter o pólo magnético rígido. Isto permitiu a avaliação da relação força/distância intermagnética. Antes da instalação do sistema magnético foi realizada uma cirurgia para a remoção do segundo molar inferior direito decíduo e exposição de parte da coroa do dente impactado. Esse procedimento foi realizado através de uma ulectomia feita com eletro bisturi para minimizar o sangramento na região e, em conseqüência, permitir uma colagem eficaz do magneto, evitando-se o descolamento durante o tracionamento. Buscou-se preservar ao máximo o folículo pericoronário do pré-molar impactado. Em seguida foi instalado um pólo magnético horizontalmente posicionado em direção ao plano oclusal (Fig. 4). Para aproximar o magneto da superfície oclusal e diminuir a distância intermagnética inicial, 


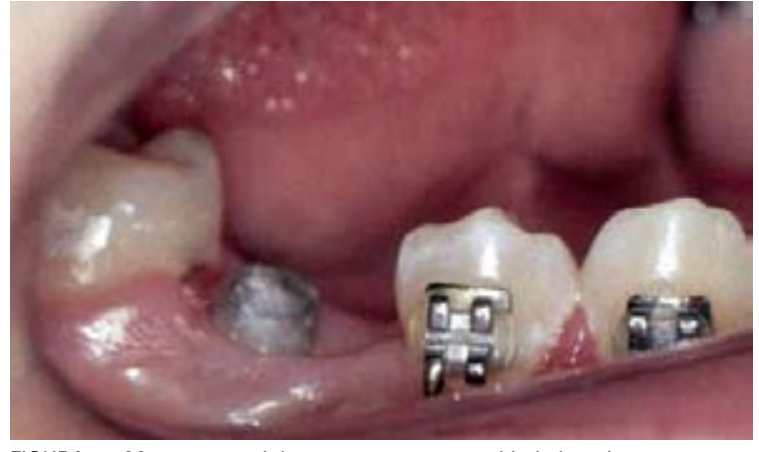

FIGURA 4 - Magneto parcialmente exposto na cavidade bucal.

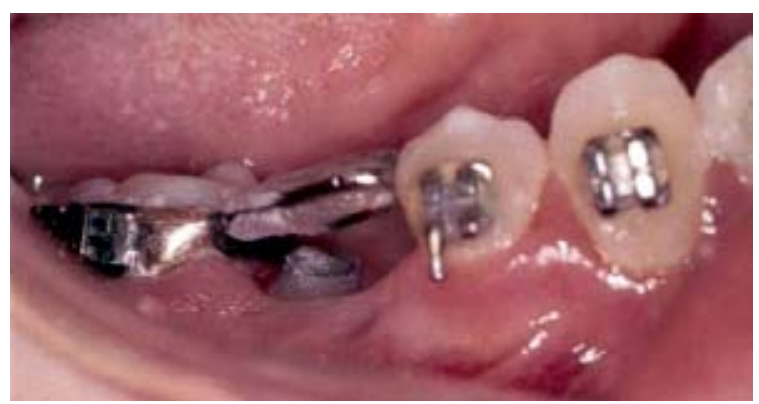

FIGURA 5 - Sistema banda-alça com o pólo magnético incrustado em seu interior.

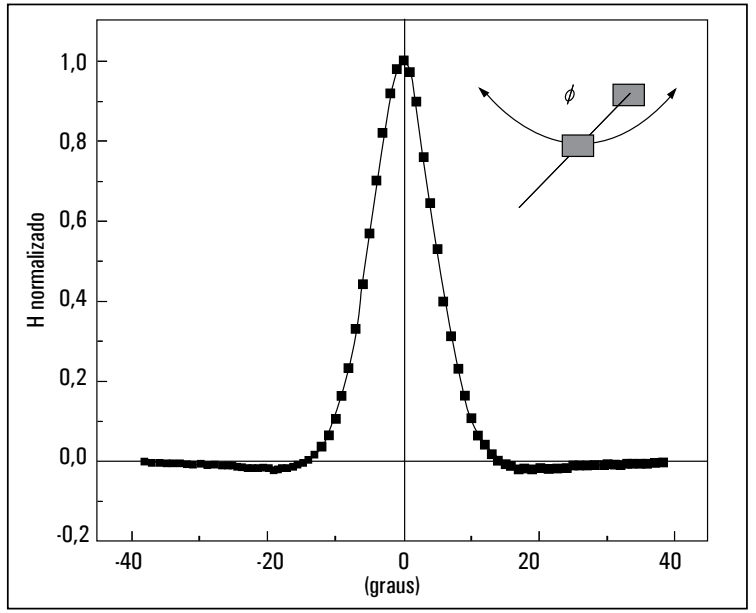

GRÁFICO 1 - Variação do campo magnético $(H)$ em função da angulação intermagnetos $\Phi$.

foi confeccionado um platô com resina fotopolimerizável (3M Single Bond ${ }^{\mathrm{TM}}$ ).

Sete dias após a cirurgia instalou-se o pólo magnético atrativo rígido apoiado no aparelho banda-alça (Fig. 5). A distância inicial intermagnetos foi de aproximadamente $2 \mathrm{~mm}$.
A paciente foi orientada a manter uma higienização adequada e não mastigar do lado do tracionamento para não danificar o sistema. Foram feitos ajustes na superfície oclusal de acrílico para evitar toques prematuros nos dentes antagonistas.

\section{RESULTADOS E DISCUSSÃO}

Através das análises de fluorescência e difração de raios $\mathrm{X}$ verificou-se que a amostra é formada por uma liga de $\mathrm{Sm}_{2} \mathrm{Co}_{17}$ contendo ainda algumas impurezas de Fe e Zr. Há também algumas indicações das fases Co e $\mathrm{Sm}_{2} \mathrm{O}_{3}$. O material apresentou uma densidade igual a $8,0 \mathrm{~g} / \mathrm{cm}^{3}$ e dureza Vickers de 598D.P.N. O material apresentou também uma magnetização igual a $(5,1 \pm 0,1) 10^{5} \mathrm{~A} / \mathrm{m}$.

O campo magnético sofre alterações tanto no plano vertical, pela alteração da quantidade de força desenvolvida em função da distância, quanto no plano horizontal pela possibilidade de inversão do campo (Gráf .1). Por isso buscou-se um melhor paralelismo entre os pólos. No entanto, o pólo magnético fixado na banda-alça foi posicionado mesialmente para que houvesse uma componente de força verticalizante do dente impactado, respeitando-se os limites de atuação do campo atrativo. Para anular uma possível inclinação do molar durante a desimpactação, toda a grade contendo o magneto e a resina acrílica foi confeccionada bem próxima à superfície distal do primeiro pré-molar.

A distância inicial entre os magnetos foi de $2 \mathrm{~mm}$, conferindo uma força de $0.50 \mathrm{~N}(1 \mathrm{~N}=101.9$ gramas força) aumentando gradualmente em função da distância, conforme comprovado por Sobrinho, Cortéz e Mohalen ${ }^{21}$.

$\mathrm{Na}$ primeira avaliação clínica e radiográfica, um mês após a instalação do sistema magnético atrativo, verificou-se que o dente tracionado se encontrava parcialmente verticalizado (Fig. 6). O platô acrílico que suportava o magneto (que antes se encontrava parcialmente recoberto pela gengiva) emergiu à cavidade bucal.

Nesta fase não houve necessidade de nenhuma ativação no sistema porque o próprio campo 


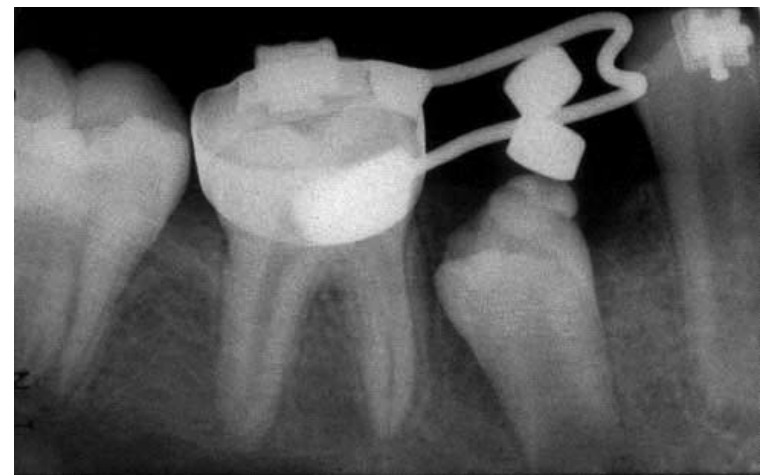

FIGURA 6 - Verticalização e extrusão parcial do segundo pré-molar inferior direito.

magnético favoreceu a extrusão dentária. Porém, com a aproximação dos pólos magnéticos o platô de acrílico foi removido e o magneto reposicionado diretamente na superfície oclusal, criando com isto uma nova ativação. Esta nova ativação forneceu aproximadamente $0.70 \mathrm{~N}$ de força de tracionamento oclusal com distância intermagnética de $1,5 \mathrm{~mm}$.

As forças magnéticas são regidas pela Lei de Coulomb $\left(\mathrm{F}=\mathrm{l} / \mathrm{d}^{2}\right)$ onde a força é inversamente proporcional ao quadrado da distância. Por isso quanto menor a distância intermagnética, maior a força de atração. No sistema mecânico convencional, no entanto, ocorre o contrário (Lei de Hooke) ${ }^{10}$.

A fase de desimpactação foi concluída em 40 dias após a implantação do sistema magnético. A coroa do dente pré-molar já se encontrava parcialmente exposta na cavidade bucal. O sistema atrativo magnético foi retirado permitindo a colagem do braquete diretamente sobre sua face vestibular. Nesta fase iniciou-se o nivelamento do dente tracionado na arcada inferior empregando um fio .012 NiTi (Fig. 7).

Após serem retirados da boca, os magnetos não apresentaram sinais de fraturas ou oxidação em nenhum dos pólos. A paciente não relatou dor, desconforto ou mobilidade excessiva durante esta fase. A ausência de desconforto e baixa mobilidade dentária também foram observadas por Graber $^{10}$. Sendo a reabsorção óssea mais rápida do que a deposição, um aumento da mobilidade dental é freqüentemente observado com o uso de forças convencionais. Apesar do movimento ser rápido, a redução na mobilidade reportada com o uso dos camposmagnéticos podeser explicada por uma taxa osteogênica pelo menos igual à taxa de reabsorção. A utilização de campos magnéticos provavelmente promove a aceleração tanto das taxas de osteogênese quanto de reabsorção.

Segundo Blechman e Steger ${ }^{5}$, o fenômeno reabsorção/osteogênese pode estar ocorrendo na membrana celular bilaminar. Através desta organela o sinal pode ser ampliado, resultando numa aceleração da fosforilação de enzimas específicas. Estas são responsáveis pela ativação de proteínas que aceleram certas funções celulares, que estavam normalmente programadas. Para os osteoblastos, por exemplo, este mecanismo poderia acelerar o padrão de osteogênese.

Inúmeras vantagens podem ser atribuídas ao sistema magnético empregado, como por exemplo, a manutenção da higidez do tecido gengival durante o tracionamento, a ausência de irritação além da fácil higienização. A ausência de fadiga do material magnético (ao contrário do observado nos materiais convencionais como elásticos, molas e fios) aliada à possibilidade de se medir previamente a força empregada e uma movimentação ortodôntica mais rápida (pela diminuição do atrito entre os materiais) também confere vantagens ao sistema. Segundo Graber $^{10}$, a relação $\mathrm{F} / \mathrm{V}$, máxima força à curta distância, orientação centrípeta tridimensional da força de atração magnética e ausência de barreira no meio bucal que possam interromper a ação da força entre os dois magnetos são características singulares dos campos magnéticos.

O método magnético de tracionamento é menos invasivo que algumas técnicas convencionais, como por exemplo o fio flexível circunferencial e a transfixação coronária. Segundo Boyd ${ }^{7}$, a primeira técnica induz reabsorção externa, anquilose, além de ocasionar a remoção excessiva de tecido ósseo. O método de transfixação, apesar de ser mais seguro com relação à ruptura dos dispositivos, 
exige um ajuste estético ao final do tratamento ortodôntico. Outro ponto positivo do método magnético é que, uma vez colado à coroa dentária e recoberto pelo retalho cirúrgico, o magneto não fica sujeito à pressão de fios ou de manipulações com elásticos.

A paciente passou a usar uma contenção ortodôntica móvel (placa de Hawley) desde então e o acompanhamento clínico é feito a cada 4 meses. Radiograficamente observou-se uma desimpactação completa do dente pré-molar que apresentou uma evolução em sua rizogênese (Fig. 7, 8). Oito meses após a conclusão dos procedimentos, 2/3 da raiz já haviam se formado, embora o ápice tenha se mantido aberto (Fig. 8). O dente apresenta uma boa implantação óssea (Fig. 9A), além de um bom posicionamento na arcada, com equilibrio funcional (Fig. 9B).

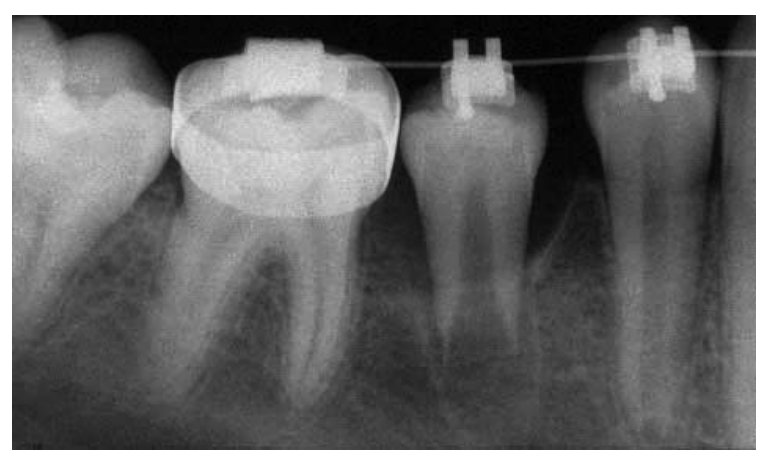

FIGURA 7 - Radiografia feita 40 dias após a desimpactação. Emprego de fio NiTi 012, para manter o dente alinhado e estável no arco.

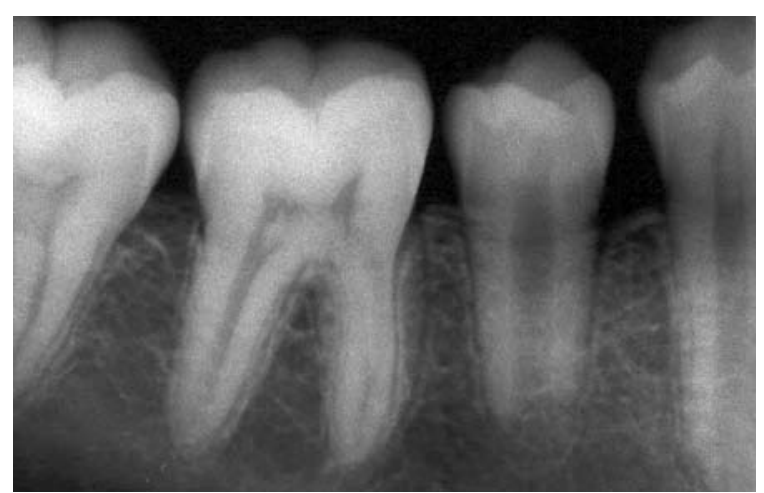

FIGURA 9A - Radiografia feita 8 meses após a desimpactação. Convém salientar a boa implantação óssea e a rizogênese em evolução.

\section{CONCLUSÃO}

A utilização da técnica magnética para desimpactação dentária, neste caso clínico, foi bastante eficaz tanto em relação ao tempo quanto em relação ao conforto proporcionado ao paciente. Os magnetos empregados geraram um campo de força contínuo e autônomo não sendo necessária a utilização de fios metálicos como guias de orientação na erupção e nem elásticos. Além do mais, o campo magnético não se isola uma vez interposto por um retalho gengival, o que propicia uma erupção guiada sem traumas para as mucosas. Esta possibilidade terapêutica poderá ser útil em desimpactações mais profundas, tais como dos caninos ectópicos, freqüentemente vistos no cotidiano.

Não obstante, para o êxito desta nova proposta

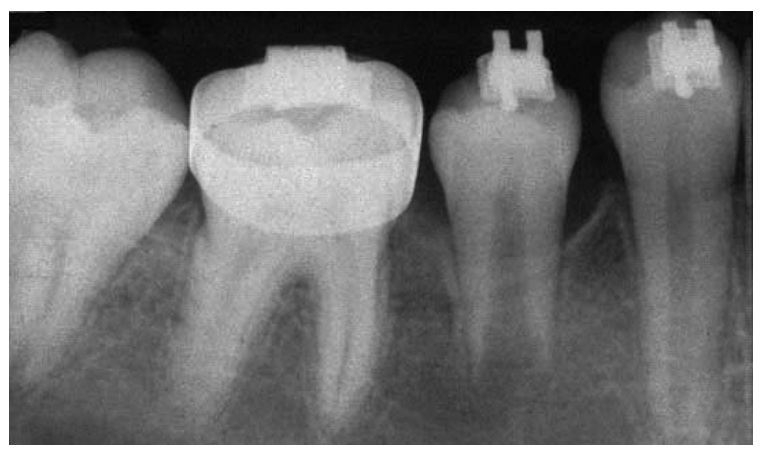

FIGURA 8 - Radiografia feita 4 meses após a desimpactação.

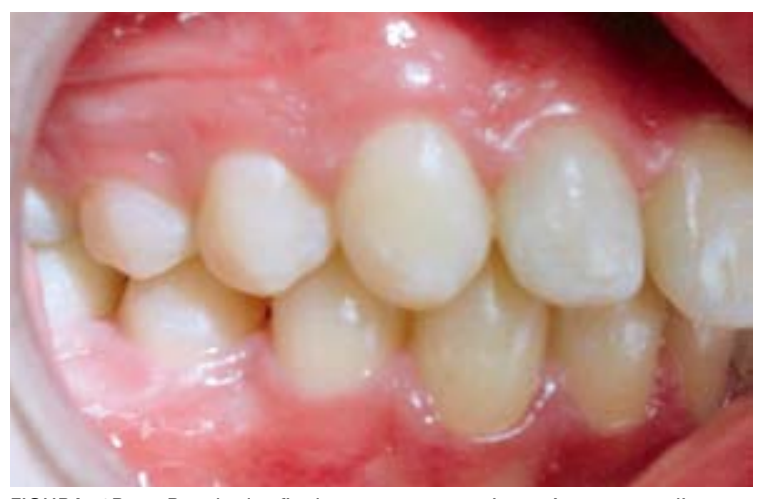

FIGURA 9B - Resultado final, um ano e meio após o procedimento. Vista lateral direita. 
terapêutica ortodôntica é necessário avançar nas pesquisas enfocando aspectos biológicos dos campos magnéticos gerados por magnetos permanentes, tanto em relação às configurações geométricas como na geração de novos materiais, permitindo a redução dos magnetos sem que haja perda nas suas propriedades.

\section{AGRADECIMENTOS}

Agradecemos à Dra. Yasmine Antonini pelo revisão final do trabalho e à Dra. Renata de Oliveira Mendes pelas sugestões e cirurgia periodontal.

\title{
A mechanical alternative with employment of magnetic force for the tooth desimpactation
}

\begin{abstract}
Aim: the present work evaluated a new mechanical alternative to guide the eruption to the oclusal plan of a impacted bicuspid tooth using attractive cobalt-samarium magnets. Methods: the treatment was accomplished in a 11 years old Class I young patient, with a impacted right inferior second premolar. The magnetic system consisted of magnets bonded to the vestibular surface of the impacted tooth and a rigid magnetic pole incrusted in a band and loop container. Previously to the installation of the magnetic system, the magnets were characterized through fluorescence analysis, $\mathrm{X}$ rays diffraction and magnetization level. The force/distance relationship used was quantified, the magnets were measured and the buccal magnetic geometry established. Results: the time used in the desimpactation was of 40 days and were necessary two magnetic activations to approach the magnets in this interval. The magnetic option in this clinical case was quite effective so much in relation to the time of treatment as in regard to the comfort proportionated to the patient. The magnets generated a continuous and autonomous force field, not being necessary to use metallic threads as orientation guides in the eruption and nor elastic. Conclusion: this therapeutic possibility will frequently be useful in such deeper desimpactation as the canine teeth ectopics, seen in the daily activity.
\end{abstract}

Key words: Magnetic force. Orthodontics. Tooth desimpactation.

\section{REFERÊNCIAS}

1. ANDREASEN, J. O. The impacted premolar. In: ANDREASEN, J. O.; PETERSEN, J. K.; LASKIN, D. M. (Ed.). Textbook and color atlas of tooth impactions: diagnosis treatment and prevention. Copenhagem: Munksgaard, 1997. p. 177-195.

2. BECKER, J. J. Permanent magnets. Sci Am, New York, v. 223, p. $92-100,1970$.

3. BIANCHI, S. D.; ROCUZZO, M. Primary impaction of primary teeth: a review and report of three cases. J Clin Pediatr Dent, Birmingham, v. 15, no. 3, p. 165-168, Spring 1991.
4. BISHARA, S. E. Impacted maxillary canines: a review. Am J Orthod Dentofacil Orthop, St. Louis, v. 101, no. 2, p.159-171, Feb. 1992.

5. BLECHMAN, A. M.; STEGER, E. R. A possible mechanism of action of repelling, molar distalizing magnets. Part.1. Am J Orthod Dentofacial Orthop, St. Louis, v. 108, no. 4, p. 428 - 431, Oct. 1995.

6. BONDEMARK, L. et al. Repelling magnets versus super elastic nickel: titanium coils in simultaneous distal movement of maxillary first and second molars. Angle Orthod, Appleton, v. 64, no. 3, p. $189-198$, May/June 1994.

7. BOYD, R. L. Clinical assessment of injuries in orthodontic movement of impacted teeth. Am J Orthod Dentofacial Orthop, St. Louis, v. 82, no. 6, p. 478-486, Dec. 1982. 
8. CENY, R. Magnets-Orthodontics: the application of magnetic forces to Orthodontics. Aust Orthod J, Brisbane, v. 5, no. 3, p. $105-113$, Mar. 1978.

9. DARENDELILER, M. A.; FRIEDLI, J. M. Treatment of an impacted canine with magnets. J Clin Orthod, Boulder, v. 28, no. 11, p. 639 - 643, Nov. 1994.

10. GRABER, T. M. Magnetos and impacted canines: north croft memorial lecture. Manchester: British Society for the Study of Orthodontics, 1998.

11. GRAZIANI, M. Cirurgia buco-maxilo-facial. 6. ed. Rio de Janeiro: Guanabara Koogan, 1976.

12. MACALISTER, A. D. An inverted lower bicuspid: report of a case. Aust J Dent, Sydney, v. 58, p. 310-311, 1956.

13. MAJOURAU, A.; NORTON, L. A. Uprighting impacted second molars with segmented springs. Am J Orthod Dentofacial Ortop, St. Louis, v. 107, p. 235-238, 1995.

14. MARTINS, D. R.; KAWAKAMI, R. Y.; HENRIQUES, J. F. C.; JANSON, G. R. P. Impacção dentária: condutas clínicas: apresentação de casos clínicos. R Dental Press Ortodon Ortop Facial, Maringá, v. 3, n. 1, p. 12-22, 1998.

15. MARZOLA, C. Retenção dental. São Paulo: Pancast, 1998

16. McDONALD, F.; YAP, W. L. The surgical exposure and application of direct traction of unerupted teeth. Am J Orthod Dentofacial Orthop, St. Louis, v. 89, p. 331-340, Apr. 1986.

17. NOMURA, Y. Inverted impaction of second premolar: two case reports. J Clin Pediatr Dent, Birmingham, v.19, no. 3, p. 205-209, 1995.
18. RAIJIC, S. MURETIC, Z; PERCAC, S. Canino impactado em crânio pré-histórico. R Dental Press Ortodon Ortop Maxilar Maringá, v. 2, n. 2, mar./abr. 1997.

19. RASMUSSEM, P.; KOTSAKI, A. Inherited primary failure of eruption in the primary dentition: report de five cases. J Dent Children, Chicago, v. 50, p. 43-47, Jan./Feb. 1999.

20. SHAPIRA, Y. et al. Bringing impacted mandibular second premolars into occlusion. J Am Dent Assoc, Chicago, v. 127, no. 7 p. 1075-1078, July 1996

21. SOBRINHO, S. I.; CORTÉZ, M. E.; MOHALEM, N. D. S. Emprego de forças magnéticas em Ortodontia: uma nova perspectiva. Anais da SBPQo - Sociedade Brasileira de Pesquisa Odontológica, 2002.

22. SOBRINHO, S. I.; CORTÉS, M.E; MOHALEM, N. D. S. Emprego de forças magnéticas em Ortodontia e Ortopedia Facial: uma revisão. R Dental Press Ortodon Ortop Facial, Maringá, v. 8 n. 4, p. 63-72, jul./ago. 2003

23. SPRINGATE, S. D.; SANDLER, P. J. Micro-magnetic retainers: an attractive solution to fixed retention. Br J Orthod, London, v. 18, no. 2, p. $139-141$, May 1991

24. VERDI, A. R. et al. Estudo clínico-radiográfico da incidência dos dentes inclusos em 3.000 indivíduos. Rev Assoc Paul Cirurg Dent, São Paulo, v. 27, n. 5, p. 274-279, set./out. 1973.

25. YUKSEL, S. et al. Magnetisch Herbeigefuhrter Durchbruch impaktierter Pramolaren bein erblich veranlagten Patienten. IOK [S.I.], v. 27, no. 2, p. 149-156, Jahrg, 1995.
Endereço para correspondência

Sisenando Itabaiana Sobrinho

Rua José Geraldo Bessa 100/102 - Nova Floresta

CEP: $31.140-390$ - Belo Horizonte/MG

Email: sise.bh@terra.com.br 